Kun-Peng Guo*

\title{
The crystal structure of $\left\{N^{1}, N^{2}\right.$-bis[2,4-dimethyl-6- (4-(tert-butyl)phenyl)(phenyl)methyl] acenaphthylene-1,2-diimino- $\mathrm{K}^{2} N, N^{\prime}$-dibromido- nickel(II) - dichloromethane(1/2), $\mathrm{C}_{64} \mathrm{H}_{64} \mathrm{Br}_{2} \mathrm{Cl}_{4} \mathrm{~N}_{2} \mathrm{Ni}$
}

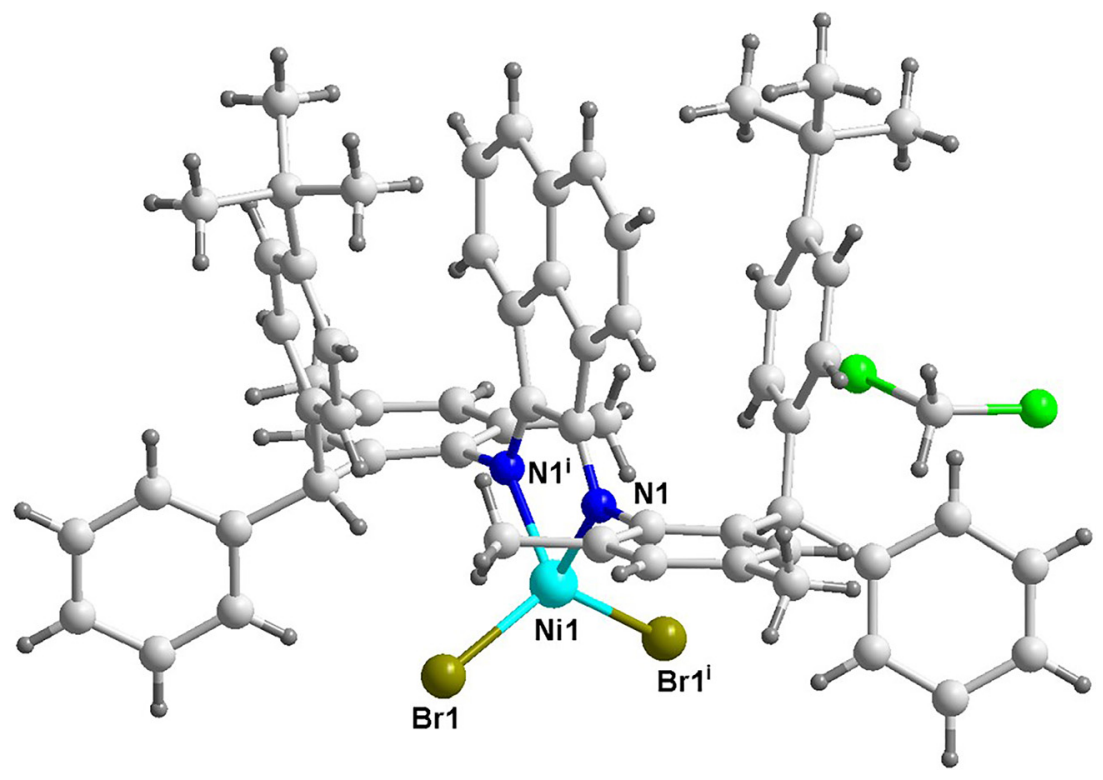

https://doi.org/10.1515/ncrs-2021-0371

Received September 25, 2021; accepted October 22, 2021;

published online November 3, 2021

Table 1: Data collection and handling.

\section{Abstract \\ $\mathrm{C}_{64} \mathrm{H}_{64} \mathrm{Br}_{2} \mathrm{Cl}_{4} \mathrm{~N}_{2} \mathrm{Ni}$, monoclinic, $C 2 / c$ (no. 15), $a=31.9723(18) \AA$, $b=11.9826(6) \AA, c=18.4722(11) \AA, \beta=125.038(2)^{\circ}$, $V=5794.4(6) \AA^{3}, Z=4, R_{g t}(F)=0.0536, w R_{r e f}\left(F^{2}\right)=0.1640$, $T=273.15 \mathrm{~K}$}

CCDC no.: 2117294

Table 1 contains crystallographic data and Table 2 contains the list of the atoms including atomic coordinates and displacement parameters.

\begin{tabular}{ll}
\hline Crystal: & Green block \\
Size: & $0.05 \times 0.04 \times 0.03 \mathrm{~mm}$ \\
Wavelength: & Mo $K \alpha$ radiation $(0.71073 \AA)$ \\
$\mu:$ & $1.94 \mathrm{~mm}^{-1}$ \\
Diffractometer, scan mode: & Bruker APEXII, $\varphi$ and $\omega$-scans \\
$\theta_{\text {max }}$, completeness: & $526.4,>99 \%$ \\
$N(h k l)_{\text {measured }}, N(h k l)_{\text {unique }}, R_{\text {int }}:$ & $16,288,5909,0.023$ \\
Criterion for $I_{\text {obs }}, N(h k l)_{\mathrm{gt}}:$ & $I_{\text {obs }}>2 \sigma\left(l_{\text {obs }}\right), 4631$ \\
$N(\text { param })_{\text {refined }}:$ & 336 \\
Programs: & Bruker programs [1], OLEX2 [2], \\
& SHELX [3, 4] \\
\hline
\end{tabular}

\section{Source of materials}

The title complex was synthesized via the one-pot reaction of acenaphthoquinone, 2-((4-(tert-butyl)phenyl)(phenyl)methyl) aniline and anhydrous $\mathrm{NiBr}_{2}$ with a molar ratio 1:2:1 from an acetic acid solution according to a reported method [5].
*Corresponding author: Kun-Peng Guo, Luohe Medical College, Luohe, Henan 462002, P. R. China, E-mail: Ihmc0395@126.com 
Table 2: Fractional atomic coordinates and isotropic or equivalent isotropic displacement parameters $\left(\AA^{2}\right)$.

\begin{tabular}{|c|c|c|c|c|}
\hline & $x$ & $y$ & $z$ & $U_{\text {iso }} * / U_{\text {eq }}$ \\
\hline $\mathrm{Br} 1$ & $0.54132(2)$ & 1.09105 (4) & $0.88296(3)$ & 0.05779 (18) \\
\hline $\mathrm{Ni1}$ & 0.500000 & $0.99384(5)$ & 0.750000 & 0.03127 (18) \\
\hline N1 & $0.45757(10)$ & $0.8660(2)$ & $0.74872(17)$ & $0.0282(6)$ \\
\hline C1 & $0.47673(12)$ & $0.7710(3)$ & $0.7510(2)$ & $0.0290(7)$ \\
\hline $\mathrm{C} 2$ & $0.46427(13)$ & $0.6540(3)$ & $0.7546(2)$ & $0.0327(7)$ \\
\hline C3 & 0.500000 & $0.5902(4)$ & 0.750000 & $0.0332(10)$ \\
\hline $\mathrm{C} 4$ & 0.500000 & $0.4725(4)$ & 0.750000 & $0.0396(11)$ \\
\hline C5 & $0.46259(16)$ & $0.4205(3)$ & $0.7561(3)$ & $0.0451(9)$ \\
\hline H5 & 0.461220 & 0.343056 & 0.756846 & $0.054^{\star}$ \\
\hline $\mathrm{C} 6$ & $0.42801(16)$ & $0.4827(3)$ & $0.7608(3)$ & $0.0480(9)$ \\
\hline H6 & 0.403724 & 0.445891 & 0.764590 & $0.058^{*}$ \\
\hline C7 & $0.42818(14)$ & $0.6011(3)$ & $0.7601(3)$ & 0.0393 (8) \\
\hline $\mathrm{H} 7$ & 0.404490 & 0.641610 & 0.763353 & $0.047^{\star}$ \\
\hline $\mathrm{C} 8$ & $0.41204(12)$ & $0.8688(3)$ & $0.7483(2)$ & $0.0294(7)$ \\
\hline C9 & $0.41792(13)$ & $0.8635(3)$ & $0.8294(2)$ & $0.0329(7)$ \\
\hline C10 & $0.37360(14)$ & $0.8622(3)$ & $0.8265(2)$ & $0.0378(8)$ \\
\hline H10 & 0.376355 & 0.859389 & 0.879406 & $0.045^{\star}$ \\
\hline C11 & $0.32520(14)$ & $0.8650(3)$ & $0.7474(3)$ & 0.0374 (8) \\
\hline C12 & 0.32137 (13) & $0.8730(3)$ & $0.6695(2)$ & $0.0338(7)$ \\
\hline H12 & 0.289157 & 0.876324 & 0.616396 & $0.041^{*}$ \\
\hline C13 & $0.36451(12)$ & $0.8763(3)$ & $0.6681(2)$ & $0.0293(7)$ \\
\hline C14 & $0.47001(15)$ & $0.8574(4)$ & $0.9156(2)$ & $0.0451(9)$ \\
\hline $\mathrm{H} 14 \mathrm{~A}$ & 0.485535 & 0.786976 & 0.919692 & $0.068^{\star}$ \\
\hline H14B & 0.466711 & 0.864585 & 0.963884 & $0.068^{*}$ \\
\hline $\mathrm{H} 14 \mathrm{C}$ & 0.490980 & 0.916829 & 0.918209 & $0.068^{\star}$ \\
\hline C15 & $0.27747(16)$ & $0.8613(4)$ & $0.7471(3)$ & $0.0540(10)$ \\
\hline H15A & 0.247852 & 0.873811 & 0.688073 & $0.081^{*}$ \\
\hline H15B & 0.279537 & 0.918268 & 0.785547 & $0.081^{\star}$ \\
\hline $\mathrm{H} 15 \mathrm{C}$ & 0.274939 & 0.789532 & 0.767334 & $0.081^{*}$ \\
\hline C16 & 0.36089 (13) & $0.8822(3)$ & $0.5820(2)$ & $0.0310(7)$ \\
\hline H16 & 0.390060 & 0.926721 & 0.594427 & $0.037^{\star}$ \\
\hline C17 & $0.31296(13)$ & $0.9431(3)$ & $0.5094(2)$ & $0.0346(7)$ \\
\hline C18 & $0.30946(16)$ & $1.0568(3)$ & $0.5187(3)$ & $0.0489(9)$ \\
\hline H18 & 0.336088 & 1.093910 & 0.568163 & $0.059^{\star}$ \\
\hline C19 & 0.26629 (19) & $1.1156(4)$ & $0.4542(3)$ & $0.0590(11)$ \\
\hline H19 & 0.264050 & 1.191795 & 0.461106 & $0.071^{*}$ \\
\hline C20 & $0.22651(16)$ & $1.0615(4)$ & $0.3796(3)$ & $0.0573(11)$ \\
\hline $\mathrm{H} 2 \mathrm{O}$ & 0.197711 & 1.100993 & 0.336444 & $0.069^{\star}$ \\
\hline C21 & $0.23001(16)$ & $0.9512(4)$ & $0.3702(3)$ & $0.0546(11)$ \\
\hline H21 & 0.203329 & 0.914780 & 0.320323 & $0.066^{\star}$ \\
\hline C22 & $0.27272(15)$ & $0.8908(3)$ & $0.4338(3)$ & 0.0449 (9) \\
\hline H22 & 0.274466 & 0.814765 & 0.425772 & $0.054^{*}$ \\
\hline C23 & $0.36703(12)$ & $0.7663(3)$ & $0.5549(2)$ & $0.0326(7)$ \\
\hline C24 & $0.33903(14)$ & $0.6760(3)$ & $0.5507(2)$ & $0.0403(8)$ \\
\hline H24 & 0.316309 & 0.684929 & 0.566364 & $0.048^{\star}$ \\
\hline C25 & $0.34420(16)$ & $0.5713(3)$ & $0.5233(3)$ & $0.0453(9)$ \\
\hline H25 & 0.324185 & 0.512216 & 0.519506 & $0.054^{\star}$ \\
\hline C26 & 0.37878 (15) & $0.5541(3)$ & $0.5015(2)$ & $0.0431(9)$ \\
\hline $\mathrm{C} 27$ & $0.40683(16)$ & $0.6454(3)$ & $0.5066(3)$ & $0.0465(9)$ \\
\hline $\mathrm{H} 27$ & 0.430344 & 0.636545 & 0.492708 & $0.056^{*}$ \\
\hline C28 & $0.40084(14)$ & $0.7503(3)$ & $0.5321(2)$ & $0.0397(8)$ \\
\hline $\mathrm{H} 28$ & 0.419861 & 0.810190 & 0.533739 & $0.048^{\star}$ \\
\hline C29 & 0.38289 (17) & $0.4404(4)$ & $0.4680(3)$ & $0.0525(10)$ \\
\hline C30 & $0.4377(2)$ & $0.4177(5)$ & $0.4979(5)$ & $0.099(2)$ \\
\hline $\mathrm{H} 30 \mathrm{~A}$ & 0.447723 & 0.472759 & 0.472806 & $0.149^{\star}$ \\
\hline
\end{tabular}

Table 2: (continued)

\begin{tabular}{lrrrr}
\hline & $\boldsymbol{x}$ & $\boldsymbol{y}$ & $\boldsymbol{z}$ & $\boldsymbol{U}_{\text {iso }} \boldsymbol{U}_{\text {eq }}$ \\
\hline H30B & 0.439677 & 0.344815 & 0.478382 & $0.149^{*}$ \\
H30C & 0.460237 & 0.421265 & 0.561215 & $0.149^{*}$ \\
C31 & $0.3644(3)$ & $0.3448(4)$ & $0.4983(5)$ & $0.096(2)$ \\
H31A & 0.382381 & 0.347240 & 0.561400 & $0.144^{*}$ \\
H31B & 0.370820 & 0.274737 & 0.481195 & $0.144^{*}$ \\
H31C & 0.328418 & 0.352762 & 0.471153 & $0.144^{*}$ \\
C32 & $0.3493(2)$ & $0.4431(4)$ & $0.3677(3)$ & $0.0677(14)$ \\
H32A & 0.314282 & 0.452744 & 0.347180 & $0.101^{*}$ \\
H32B & 0.352843 & 0.374261 & 0.345198 & $0.101^{*}$ \\
H32C & 0.359434 & 0.504119 & 0.347357 & $0.101^{*}$ \\
Cl1 & $0.40937(10)$ & $0.8178(2)$ & $0.3333(2)$ & $0.1404(9)$ \\
Cl2 & $0.29885(8)$ & $0.84487(15)$ & $0.20692(13)$ & $0.1120(6)$ \\
C33 & $0.3463(3)$ & $0.8355(6)$ & $0.3130(5)$ & $0.108(2)$ \\
H33A & 0.339895 & 0.772661 & 0.338530 & $0.129^{*}$ \\
H33B & 0.346742 & 0.902481 & 0.342960 & $0.129^{*}$ \\
\hline
\end{tabular}

The crystals of the title complex were recrystallized from the dichloromethane solution after three day. Yield 64\% (based on acenaphthoquinone).

\section{Experimental details}

The structure was solved by direct methods with the SHELXS-2018 program. All $\mathrm{H}$-atoms from $\mathrm{C}$ atoms were positioned with idealized geometry and refined isotropic $\left(U_{\text {iso }}(\mathrm{H})=1.2 U_{\text {eq }}(\mathrm{C})\right)$ using a riding model with $\mathrm{C}-\mathrm{H}=0$. $96 \AA$ for tert-butyl groups, $\mathrm{C}-\mathrm{H}=0.93 \AA$ for phenyl groups, $\mathrm{C}-\mathrm{H}=0.98 \AA$ for $\mathrm{C} 16-\mathrm{H} 16$, and $\mathrm{C}-\mathrm{H}=0.97 \AA$ A for dichloromethane, respectively.

\section{Comment}

$N, N^{\prime}$-bis(2,4,6-trimethylphenyl)acenaphthen-1,2-diimine and its derivatives have been synthesized as useful ligands to exploit a/-diimine nickel catalysts for various olefin polymerization reactions [5-16]. It is interesting that the substituent groups of these diimines affected the catalytic performance greatly. Therefore, people have a strong interest in the selection of substituent groups. Thus, we here report the single crystal structure of a new acenaphthen1,2-diimine-based Ni(II) complex.

The title complex belongs to the acenaphthen-1,2-diimine based Ni(II) complexes. The asymmetric unit consists of one half of a ligand, one half of a $\mathrm{Ni}(\mathrm{II})$ cation, one bromide and one crystal dichloromethane molecule. The $\mathrm{Ni}(\mathrm{II})$ cation adapts an distorted tetrahedral geometry by coordinating two $\mathrm{N}$ atoms from the ligand and two bromido ligands. The bond lengths of Ni-N and Ni-Br are 2.037 and 2.326 $\AA$, respectively, 
which are comparable with the values of the reported results [5-17].

Acknowledgments: We thank the 2019 Innovation and Entrepreneurship Development Ability Improvement Engineering Scientific Research Project of Luohe Medical College (2019-LYZKYYB028).

Author contributions: The author has accepted responsibility for the entire content of this submitted manuscript and approved submission.

Research funding: None declared.

Conflict of interest statement: The author declares no conflicts of interest regarding this article.

\section{References}

1. Bruker. SAINT (v6.45A); Bruker AXS Inc: Madison, Wisconsin, USA, 2003.

2. Dolomanov O. V., Bourhis L. J., Gildea R. J., Howard J. A. K., Puschmann H. OLEX2: a complete structure solution, refinement and analysis program. J. Appl. Crystallogr. 2009, 42, 339-341.

3. Sheldrick G. M. Crystal structure refinement with SHELXL. Acta Crystallogr. C: Struct. Chem. 2015, C71, 3-8.

4. Sheldrick $\mathrm{G}$. Using phases to determine the space group. Acta Crystallogr. A 2018, A74, A353.

5. Maldanis R. J., Wood J. S., Chandrasekaran A., Rausch M. D., Chien J. C. W. The formation and polymerization behavior of $\mathrm{Ni}(\mathrm{II})$ $\alpha$-diimine complexes using various aluminum activators.

J. Organomet. Chem. 2002, 645, 158-167.

6. Cherian A. E., Lobkovsky E. B., Coates G. W. Chiral anilines: development of C2-symmetric, late-transition metal catalysts for isoselective 2-butene polymerization. Chem. Commun. 2003, 39, 2566-2567.

7. Jin G., Zhang D. Micron-granula polyolefin with self-immobilized nickel and iron diimine catalysts bearing one or two allyl groups. J. Polym. Sci, Part A: Polym. Chem. 2004, 42, 1018-1024.
8. Yuan J., Zhang Z., Xu W., Zhao J., Mu Y., Chen J. \{Bis[N, $N^{\prime}$-(2-alkyl6-para-methylphenyl)phenyl)imino]acenaphthene\} dibromonickel catalysts bearing bulky methylphenyl groups: synthesis, characterization, crystal structures and application in catalytic polymerization of ethylene and styrene. Transition Met. Chem. 2014, 39, 769-779.

9. Wang F., Tanaka R., Cai Z., Nakayama Y., Shiono T. Synthesis of highly branched polyolefins using phenyl substituted $\alpha$-diimine $\mathrm{Ni}(\mathrm{II})$ catalysts. Polymers 2016, 8, 160-165.

10. Lian K., Zhu Y., Li W., Dai S., Chen C. Direct synthesis of thermoplastic polyolefin elastomers from nickel-catalyzed ethylene polymerization. Macromolecules 2017, 50, 6074-6080.

11. Wang F., Tanaka R., Li Q., Nakayama Y., Shiono T. Chain-walking polymerization of linear internal octenes catalyzed by a/diimine nickel complexes. Organometallics 2018, 37, 1358-1367.

12. Fang J., Sui X., Li Y., Chen C. Synthesis of polyolefin elastomers from unsymmetrical a/-diimine nickel catalyzed olein polymerization. Polym. Chem. 2018, 9, 4143-4149.

13. Pei L., Liu F., Liao H., Gao J., Zhong L., Gao H., Wu Q. Synthesis of polyethylenes with controlled branching with a/-diimine nickel catalysts and revisiting formation of long-chain branching. ACS Catal. 2018, 8, 1104-1113.

14. Guo L., Lian K., Kong W., Xu S., Jiang G., Dai S. Synthesis of various branched ultra-high-molecular-weight polyethylenes using sterically hindered acenaphthene-based a/-diimine Ni(II) catalysts. Organometallics 2018, 37, 2442-2449.

15. Tian S., Zhang Y., Li R., Wang F., Li W. Cationic para-benzhydryl substituted a/-diimine nickel catalyzed ethylene and 1-decene polymerizations via controllable chain-walking. Inorg. Chim. Acta. 2019, 486, 492-498.

16. Dai S., Li S., Xu G., Wu C., Liao Y., Guo L. Flexible cycloalkyl substituents in insertion polymerization with a/-diimine nickel and palladium species. Polym. Chem. 2020, 11, 1393-1400.

17. Du S., Xing Q., Flisak Z., Yue E., Sun Y., Sun W-H. Ethylene polymerization by the thermally unique 1-[2-(bis(4-fluoro phenyl) methyl)-4,6-dimethylphenylimino]-2-aryliminoacenaphthylnickel precursors. Dalton Trans. 2015, 44, 12282. 